OPEN ACCESS

Edited by:

Ozgur Kasapcopur:

Istanbul University-Cerrahpasa, Turkey

Reviewed by:

Randy Q. Cron,

University of Alabama at Birmingham,

United States

Fatih Haslak,

Istanbul University Cerrahpasa, Turkey

*Correspondence:

Angelo Ravelli

angeloravelli@gaslini.org

Specialty section: This article was submitted to Pediatric Rheumatology, a section of the journal

Frontiers in Pediatrics

Received: 15 March 2021 Accepted: 12 May 2021 Published: 04 June 2021

Citation: Matucci-Cerinic C, Caorsi R, Consolaro A, Rosina S, Civino A and

Ravelli A (2021) Multisystem Inflammatory Syndrome in Children: Unique Disease or Part of the

Kawasaki Disease Spectrum?

Front. Pediatr. 9:680813.

doi: 10.3389/fped.2021.680813

\section{Multisystem Inflammatory Syndrome in Children: Unique Disease or Part of the Kawasaki Disease Spectrum?}

\author{
Caterina Matucci-Cerinic ${ }^{1}$, Roberta Caorsi ${ }^{2}$, Alessandro Consolaro ${ }^{1,2}$, Silvia Rosina ${ }^{2}$, \\ Adele Civino ${ }^{3}$ and Angelo Ravelli ${ }^{1,2,4 *}$
}

'Dipartimento di Neuroscienze, Riabilitazione, Oftalmologia, Genetica e Scienze Materno-Infantili, Università degli Studi di Genova, Genoa, Italy, ${ }^{2}$ Unità Operativa Complessa (UOC) Clinica Pediatrica e Reumatologia, Istituto di Ricovero e Cura a Carattere Scientifico (IRCCS) Istituto Giannina Gaslini, Genoa, Italy, ${ }^{3}$ Unità di Reumatologia e Immunologia Pediatrica,

Ospedale Vito Fazzi, Lecce, Italy, ${ }^{4}$ Sechenov First Moscow State Medical University, Moscow, Russia

One of the most intriguing and mysterious phenomena observed during the COVID-19 pandemic has been represented by the occurrence of the multisystem inflammatory syndrome in children and adolescents (MIS-C). Patients with this condition have some overlapping signs and symptoms with those of Kawasaki disease (KD), but also display clinical features that are uncommon or less frequent in this illness, such as diarrhea, abdominal pain and myocardial involvement. The sickest patients may develop multiorgan failure and shock, usually due to myocarditis. Management is based on the administration of intravenous immunoglobulin, glucocorticoids and, in the most severe instances, anakinra. It is still debated whether MIS-C and KD represent different illnesses or are part of the same disease spectrum. The aim of the present review is to analyze critically the evidence in favor of the latter hypothesis and to provide the authors' personal interpretation of the relationship between the two conditions.

Keywords: Kawasaki disease, multisystem inflammatory syndrome in children, COVID-19, MIS-C, SARS-CoV-2, toxic shock syndrome, macrophage activation syndrome

\section{INTRODUCTION}

One of the most challenging and enigmatic phenomena observed during the COVID 19 pandemic has been the emergence of the multisystem inflammatory syndrome in children (MIS-C) (1-7). The presenting signs and symptoms were a mix of those of Kawasaki disease (KD) and toxic shock syndrome (TSS), and were characterized, among others, by fever, gastrointestinal complaints, and cardiac involvement. A number of these children required urgent intensive care treatment due to the development of multiorgan failure and circulatory shock, usually of myocardial origin. Some had evidence of macrophage activation syndrome (MAS). Laboratory abnormalities included markedly elevated acute phase reactants, increased ferritin and D-dimer, hypoalbuminemia as well as lymphopenia and relative thrombocytopenia. Patients with myocarditis had elevated levels of pro-B-type natriuretic peptide (proBNP) and troponin. Management was based on the administration of intravenous immunoglobulin (IV Ig) and glucocorticoids. In some instances, IL-1, IL-6 or tumor necrosis factor inhibitors were given. A temporal association with SARS-CoV2 infection has been hypothesized because some children tested positive for the virus, either by reverse transcriptase-polymerase chain reaction (RT-PCR) or serology, or were exposed to potential contact with a household member affected with COVID-19. 
The severity of this condition contrasted with the initial reports from China and Western countries, which had shown that relatively few children and adolescents were affected by COVID-19, and that most of those infected had experienced milder disease compared to adults (8-10). However, epidemiologic data indicated that the onset of MIS-C occurred 38 weeks after prior infection or known exposure, suggesting that SARS-CoV-2 acted as a trigger of a post-infectious inflammatory process (likely driven by some aspect of the adaptive immune system) $(2,11)$. Indeed, the vast majority (>80-90\%) of children are positive for antibodies to SARS-CoV-2, whereas a smaller subset may be positive on RT-PCR for the virus. However, based on high cycle thresholds to detect the virus by RT-PCR in MIS-C patients, it is likely the virus is no longer infectious. In parallel with the rise in the number of articles describing the features of this condition in various parts of the world, an intense debate began regarding whether MIS-C and KD represent different illnesses with overlapping clinical features or are on the same disease spectrum. Although most experts favor the assumption that MIS-C is a novel entity with respect to $\mathrm{KD}(4,11-17)$, some, including us, have argued that the two disorders may be a continuum, with some of the differences in phenotypic severity being due to the magnitude or kinetics of the immune response (18).

In the present viewpoint, we examine critically the evidence that supports the latter hypothesis and provide our interpretation of the relationship between the two conditions.

\section{RELATIONSHIP BETWEEN KD AND SARS-COV-2}

During COVID-19 pandemic a large number of children with classic or incomplete KD by the American Heart Association (AHA) criteria (19) were seen in affected countries. Many of these cases appeared to be related to SARS-CoV-2 infection, as they tested positive on either RT-PCR or serology. In Italy, most instances of $\mathrm{KD}$ occurred during the lockdown period imposed by public health authorities to contain the spread of the epidemic. Because in these months (March-May 2020) children stayed at home and neither attended school nor had social interactions with peers, they were likely not exposed to infectious agents other than the SARS-CoV-2. It is, thus, conceivable that at least a fraction of the cases of "genuine" KD seen during the pandemic were linked to SARS-CoV-2, as it has been ascertained for MISC. Furthermore, considering that KD has long been thought to be related to an infectious and/or environmental trigger, albeit still elusive (20), if one admits that SARS-CoV-2 did not play a causative role, a profound drop in the prevalence of KD should have been expected during the lockdown, owing to the lesser overall infectious morbidity in the pediatric population.

\section{CLINICAL SIMILARITIES BETWEEN KD AND MIS-C}

Although most children with MIS-C in the reported series did not fulfill the AHA criteria for $\mathrm{KD}$, all had persistent fever and a variable proportion displayed one or more of the typical clinical manifestations of $\mathrm{KD}$, namely rash, conjunctivitis, lips or oral changes, erythema/edema of the extremities, or cervical lymphadenopathy. Notably, the majority of children meeting the case definition for MIS-C seen at authors hospital had conjunctival injection that spared the limbus, a sign characteristic of KD (19). These observations underscore the presence of many similarities in the clinical phenotype of the two conditions.

One of the arguments that are put forward to support the diversity between the two illnesses is that children with MIS-C have a high frequency of signs and symptoms that are unusual or occur rarely in $\mathrm{KD}$, especially abdominal pain, vomiting, diarrhea, myocardial injury, and signs of meningeal irritation. However, all these features can be seen in classic KD. Vomiting, diarrhea and abdominal pain are part of the gastrointestinal symptoms of $\mathrm{KD}$, together with hepatitis and hydrops of the gallbladder (16). Myocardial dysfunction occurs frequently in acute $\mathrm{KD}$, and myocardial inflammation has been documented by scintigraphy in $50-70 \%$ of cases $(19,21)$. In addition, during the acute stage of KD, electrocardiography may show sinus and atrioventricular node functional abnormalities, with prolonged PR interval and non-specific ST and T-wave changes or low voltage (19). Note that pro-BNP and serum troponin, used in MIS-C to assess the severity of myocarditis, have been proposed as useful markers of cardiac involvement in $\operatorname{KD}(22,23)$. Extreme irritability, exceeding that observed in other febrile illnesses, and aseptic meningitis are common neurologic findings of $\mathrm{KD}$ $(19,24)$. The development of coronary artery dilatation or aneurysms in some patients with MIS-C is difficult to reconcile with the hypothesis of the diversity of the two illnesses, as this complication has previously been attributed only to $\mathrm{KD}$ in pediatric patients.

As compared with $\mathrm{KD}$, MIS-C is marked by more intense inflammation and by the frank tendency toward the development of shock and, to a lesser extent, MAS. However, TSS, which is seen in around $5 \%$ of children with $\mathrm{KD}$ (25), has many aspects in common with the shock syndrome of MIS-C. The development of MAS has been reported in 1-2\% of cases of KD, but it is thought to be underrecognized (26). Thrombocytopenia is another feature of MIS-C that is not typical of $\mathrm{KD}$, which is characterized by thrombocytosis. However, a drop in platelet count is frequently encountered in both KD-associated TSS and MAS. A further distinctive hematologic abnormality of MIS-C is lymphopenia, which is usually not observed in $\mathrm{KD}$, and is a hallmark of severe COVID-19, although its pathophysiology is unclear (27). Hyponatremia, rhabdomyolysis, and image findings of corpus callosum inflammation can also occur in MIS-C.

There are several similarities in the therapeutic approach to MIS-C and KD. A high proportion (70-80\%) of children with MIS-C have been treated initially with IVIG. Because this therapeutic intervention is part of the standard protocol for $\mathrm{KD}$, its choice implies that many physicians who first saw these patients had the clinical impression of $\mathrm{KD}$, although the choice of this intervention could be due to the aim to control a potentially infectious process or to possibly prevent acquired coronary artery changes. In case of non-response to IVIG, shock or organthreatening disease, adjunctive therapy with glucocorticoids was 
TABLE 1 | Main similarities and differences between MIS-C and KD.

Clinical manifestations common to both KD and MIS-C

Fever, skin rash, conjunctival injection, cervical adenopathy, lip and oral changes, swollen hands and feet, irritability

Clinical manifestations frequent in MIS-C, but less common in KD Abdominal pain, diarrhea, meningeal signs, myocarditis, MAS $(1-2 \%$ in $\mathrm{KD}$, but $20-30 \%$ in MIS-C), toxic shock syndrome (5-7\% in KD, but $30-40 \%$ in MIS-C)

Laboratory abnormalities seen in MIS-C, but not in KD

Lymphopenia, relative thrombocytopenia (with the exception of MAS and TSS, in which thrombocytopenia is frequent)

Other similarities between MIS-C and KD

The vast majority of children with MIS-C were given initial treatment with IVIG Glucocorticoids were effective in patients with IVIG resistance, myocarditis or major complications (TSS or MAS)

The IL-1 inhibitor anakinra is used in severe instances of both MIS-C and KD Both MIS-C and KD pursue a self-limited course, with recovery within 2-3 weeks Some children with MIS-C developed coronary aneurysms

Both MIS-C and KD occurred during the lockdown, in the spring of 2020, when children were likely not exposed to infectious agents other than SARS-CoV-2* After the end of May 2020, after the abate of COVID-19 epidemic, MIS-C and KD disappeared simultaneously*

The second wave of COVID-19, in the fall of 2020, was accompanied by a resurgence of both MIS-C and KD*

Main difference between MIS-C and KD

Children with MIS-C are older than those with $\mathrm{KD}$ (median age in MIS-C > 5 years vs. $<5$ years in KD)

MIS-C, multisystem inflammatory syndrome in children; KD, Kawasaki disease; TSS, toxic shock syndrome; MAS, macrophage activation syndrome; IVIG, intravenous immunoglobulin.

* Observation made in Italy.

usually given (28), which is analogous to the regimens proposed for IVIG-refractory $\operatorname{KD}(19,29)$. The IL-1 inhibitor anakinra has been occasionally employed for treatment of MIS-C resistant to IVIG and glucocorticoids or impending MAS. This biologic medication is becoming increasingly more popular also for the management of KD after failure of IVIG and its efficacy in KD is being scrutinized in a phase IIa trial (30).

A further similarity between the two condition is the selflimited clinical course, which usually lasts 2-3 weeks. Recently, with improved recognition and treatment of MIS-C, the average hospital stay, even for patients presenting with shock, has decreased to nearly 5 days (31). Finally, the parallelism between MIS-C and KD has been underscored in the experience in Italy by their simultaneous disappearance after the regress of the pandemic, at the end of May 2020, and by their concurrent reemergence around 1 month after the resurgence of COVID-19, in the fall of 2020 .

The main similarities and differences between KD and MIS-C are summarized in Table $\mathbf{1 .}$

\section{WHY THE AGE OF CHILDREN WITH MIS-C IS HIGHER THAN THAT OF CHILDREN WITH KD?}

A feature that is pointed to as distinguishing MIS-C as a unique disease process vs. KD is that the median age of MIS-C cases was 9-10 years in the largest reported series $(1-6,32)$, whereas KD occurs predominantly in children 5 years of age or younger and has a peak incidence at around 10 months of age (33). There are, however, several reasons that may explain why younger children are more spared than older children and adolescents by COVID-19, in general, and, as a consequence, also by MIS-C.

In the first years of life, the immune system may be more "trained" to fight against viral infections owing to repeated vaccination procedures $(34,35)$. Notably, an amino acid sequence homology between glycoprotein components of SARS-CoV-2 and measles and rubella viruses has been identified. Using an antibody epitope prediction online tool, the homologous sequence appeared to have an epitope property and to be involved in antibody production. These findings have led to suggest that humoral immunity created through the measles, mumps and rubella (MMR) vaccine could provide a defense against COVID19 (36). Younger children can also be protected against SARS$\mathrm{CoV}-2$ in virtue of a cross-reactive immunity induced after the encounter with other coronaviruses, which are a frequent cause of respiratory tract infections in preschool age.

Another potential explanation for the lower rates of SARS$\mathrm{CoV}-2$ infection in children is the lower expression of the cell surface enzyme angiotensin-converting enzyme 2 (ACE2), a receptor that has been proven to bind to SARS-CoV-2 spike protein and to promote internalization of the virus into human cells (37). In a recent study, children aged 4-9 years were found to have lower gene expression of ACE2 in nasal epithelial samples compared with older children, young adults, and adults (38). It should be recognized, however, that the vast majority of children infected with SARS-CoV-2 do not develop MIS-C irrespective of previous vaccination with MMR and that the lower expression of ACE2 may explain the lesser severity of COVID-19, but does not explain why some children develop MIS-C and some do not.

Notably, a recent study has suggested that ACE2 expression decreases with aging (39). It could be postulated that if an individual has higher ACE2 expression, even if the virus blocks ACE2 via binding, the amount of remaining ACE2 might still be significant. Given the antinflammatory role of ACE2, its higher expression may thus, provide protection against MIS-C.

The distinctive lesser susceptibility to develop MIS-C in the early ages might also be secondary to the immaturity of the immune system, which may be less able to mount a hyperinflammatory response or a cytokine storm syndrome (40).

Epidemiologic studies of MIS-C suggest that younger children are more likely to present with KD-like features, while older children are more likely to develop gastrointestinal symptoms (severe abdominal pain, vomiting, diarrhea), myocarditis and shock, and may be more likely to present with the features of MAS $(17,41)$. Notably, children presenting on the severe spectrum of classic KD with TSS or MAS are usually older and are boys, consistent with the demographic described in MIS-C (18). The coronary changes, when seen, seem also more likely to develop in the younger KD-like group.

\section{A UNIQUE DISEASE SPECTRUM?}

Of the 149 cases of inflammatory illness in children and adolescents registered in Italy between February and May 2020 
(32), 96 met the AHA criteria for classic or incomplete KD, 10 met the same criteria plus the case definition for MIS-C, and another 43 only met only the case definition for MIS-C. The clinical diversities between patients with MIS-C and KD paralleled those observed in other series, and children with MIS-C tested positive for SARS-CoV-2 more frequently that those with KD. However, the occurrence of the two illnesses in the same population, during the same period of time, and when children were exposed to virtually no infectious agent other than the SARS-CoV-2 due to the lockdown confinement suggests, on epidemiological grounds, that they represent a disease continuum, with $\mathrm{KD}$ being at the more benign end of the spectrum and MIS-C at the most severe end.

\section{PROPOSED COMMON PATHOPHYSIOLOGY OF KD AND MIS-C}

The etiology of KD is unknown, but it is generally considered the consequence of an abnormal immune response, in genetically predisposed children, to infectious triggers entering through the upper respiratory tract. Multiple infectious agents have been suspected over the years, including respiratory RNA virus. In 2005, a group from New Haven (CT, USA) detected a novel human coronavirus, named New Haven coronavirus (HCoV$\mathrm{NH}$ ), in the respiratory secretions of 8 of 11 children with $\mathrm{KD}$ vs. 1 of 22 controls tested by RT-PCR. A serological test was not performed (42). Another study made in Japan evaluated the association between two different coronaviruses (HCoVNL63 and HCoV-229E) and KD through serological tests. No difference in HCoV-NL63 antibody positivity was found between patients and controls on immunofluorescence assay, whereas antibody level for $\mathrm{HCoV}-229 \mathrm{E}$ was higher in patients with $\mathrm{KD}$ (43). Although the association between $\mathrm{KD}$ and the coronavirus family has not been confirmed in subsequent studies (19), these observations appear intriguing in the light of the possible relationship between KD and SARS-CoV-2.

As highlighted elsewhere (44), autoinflammatory diseases have taught us that many rheumatic conditions may represent syndromes rather than diseases. A variety of monogenic illness have, indeed, been found to mimic the clinical features of polyarteritis nodosa $(45,46)$, Behçet disease (47), rheumatoid factor positive polyarthritis (48), systemic juvenile idiopathic arthritis (49) and systemic lupus erythematosus (50). These observations indicate that many conditions that have been traditionally called "diseases" are actually "syndromes," whose pathophysiology may be exemplified as a sort of funnel, that is, as a stereotyped way of reacting to multiple different etiologic factors in individuals possessing a particular genetic predisposition. The recognition of the etiology may be of paramount relevance for the treatment, as demonstrated by the dramatic effectiveness of anti-tumor necrosis factor agents in polyarteritis nodosa associated with ADA2 mutation (51).

In our view, the funnel model may be well-suited to illustrate the common pathophysiology of KD and MIS-C (Figure 1). In the case of MIS-C, an extremely aggressive and invasive virus like SARS-CoV-2, which has shown the capacity to cause a
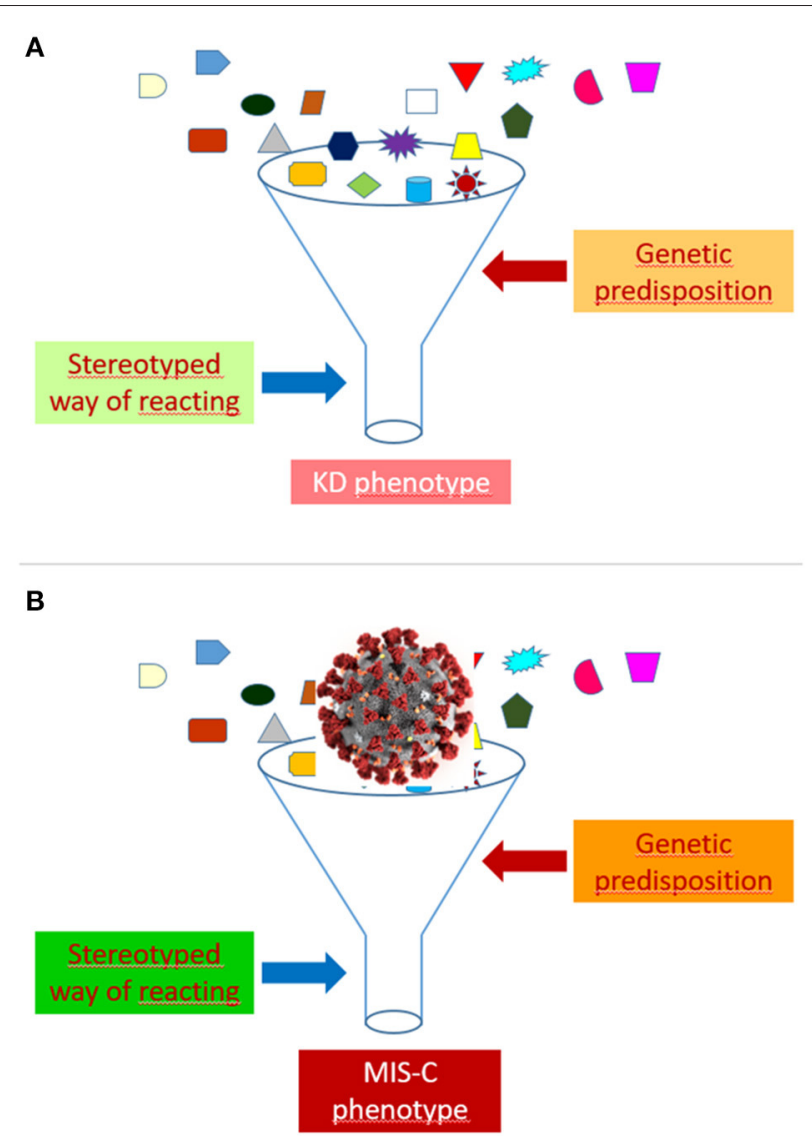

FIGURE 1 | Funnel model of the pathophysiology of Kawasaki disease (KD) (A) and multisystem inflammatory syndrome in children (MIS-C) (B).

cytokine storm syndrome in adults with COVID-19 pneumonia, could induce, when presents with a massive viral load, a clinical phenotype much more inflammatory and acute than that of $\mathrm{KD}$, and marked, in addition to the typical manifestations of $\mathrm{KD}$, by a higher frequency of less common or atypical disease manifestations and of serious complications, such as myocarditis, TSS and MAS.

The development of a KD or a MIS-C phenotype after the contact with SARS-CoV-2 might depend on several factors, including, but not limited to, viral load, virulence of viral strains, child age, intensity or kinetics of the immune response, ethnic or socio-economic factors, comorbidities (especially obesity), and genetic background. Differences between patients in the viral burden could account for the more frequent positivity of SARSCoV-2 tests in more severe cases.

Henoch-Schönlein purpura (HSP), the most common vasculitis in children, is also a syndrome. It is often preceded by a respiratory tract infection and multiple case studies have suggested a correlation with virtually all respiratory pathogens (52). Although usually benign, it can occasionally cause severe involvement of the kidney, gastrointestinal tract and central nervous system. Like KD, the pathophysiology of HSP appears consistent with an abnormal and stereotyped immune reaction to an infectious agent in genetically predisposed individuals. 
Although rather different, HSP has a self-remitting course, like KD.

Although the genetic determinants of KD are still elusive, that susceptibility is shaped by genetic influences is suggested by the preferential involvement of males, the predilection for particular racial/ethnic groups, with an incidence in Japan, Chorea and Taiwan 10- to 20-fold higher than in the United States, and the observation of an increased risk in family members and twins (19). The hypothesis of a common predisposing background between KD and MIS-C is corroborated by the report that 2 of 28 patients with MIS-C had KD in the past (53).

The role of genetic determinants in the induction of MIS-C vs. $\mathrm{KD}$ is highlighted by the marked epidemiologic differences among ethnic groups. In the United States, MIS-C had a greater impact on children of Afro-American and Hispanic ethnicity (5, 6) as in France and UK, were children of Afro-Caribbean descent were particularly hit $(1,3,54)$. Conversely, MIS-C was apparently not observed in Japan and Chorea, countries characterized by a markedly elevated prevalence of KD. Despite a very high impact of COVID-19, MIS-C was not reported in China. These issues may lead to speculate that subjects belonging to ethnic groups less affected by classic KD may be distinctly susceptible to develop a more aggressive phenotype of KD, including MIS-C.

That a shared genetic background may underlie a continuum of inflammatory disorders of varied severity has been suggested by the detection of genetic similarities among recurrent aphthous stomatitis, periodic fever, aphthous stomatitis, pharyngitis, and cervical adenitis (PFAPA) syndrome, and Behçet disease. The genotypic overlap places these disorders on a common spectrum, with recurrent aphthous stomatitis on the mild end, Behçet disease on the severe end, and PFAPA intermediate (55).

Recent studies suggest that a defective antiviral response may be contributory in some patients with COVID-19. Inborn errors of type I interferon immunity and auto antibodies against type I interferons have been discovered in the most severe cases of COVID-19 $(56,57)$. It is, thus, conceivable that host immune dysregulation, as well as a molecular mimicry between SARS$\mathrm{CoV}-2$ and self-antigens, may be involved in the induction of the severe inflammatory manifestations of MIS-C.

The inflammatory response in MIS-C was found to share several features with $\mathrm{KD}$, but also to differ from this condition in the proportion of particular subsets of T-lymphocytes, the characteristics of IL-17A-mediated immunopathology, the concentration of biomarkers of arterial inflammation and damage, and the profile of autoantibodies to proteins involved

\section{REFERENCES}

1. Riphagen S, Gomez X, Gonzalez-Martinez C, Wilkinson N, Theocaris P. Hyperinflammatory shock in children during COVID-19 pandemic. Lancet. (2020) 395:1607-8. doi: 10.1016/S0140-6736(20)31094-1

2. Verdoni L, Mazza A, Gervasoni A, Martelli L, Ruggeri M, Ciuffreda M, et al. An outbreak of severe Kawasaki-like disease at the Italian epicentre of the SARS-CoV-2 epidemic: an observational cohort study. Lancet. (2020) 395:1771-8. doi: 10.1016/S0140-6736(20)31103-X in immune response or to structural components of heart and blood vessels (58). However, the meaning of this study is affected by the choice of contrasting children with MIS-C with a historical cohort of KD seen before COVID-19 pandemic. In our view, the comparison of patients with the features of MIS-C and KD seen during the course of the pandemic could provide better insights into the relationship between the two conditions, particularly in the light of their common relationship with SARS-CoV-2.

\section{CONCLUSIONS AND FUTURE DIRECTIONS}

Based on the above considerations, we favor the hypothesis that MIS-C is on the KD spectrum, instead of representing a new childhood inflammatory disorder separate from $\mathrm{KD}$. The occurrence of a KD-like condition in association with SARS Cov2 infection underscores the notion that $\mathrm{KD}$ is not a disease, but rather a syndrome, whose main features and phenotypic severity depend on the magnitude and type of the immune response as well as on the characteristics of the host and of the triggering infectious agent (44). Notably, the interpretation of $\mathrm{KD}$ as a syndrome is in keeping with the first description by Tomisaku Kawasaki, who called it "acute febrile mucocutaneous lymph node syndrome" $(59,60)$. It should be recognized, however, that there are still limited data on MIS-C, particularly regarding well-established diagnostic criteria, pathophysiology, and outcome information. Thus, further studies of the genetic and immunopathologic background are required to establish more precisely the relationship between MIS-C and KD. More in general, the spectrum of pathology that has emerged during the pandemic offers a unique opportunity for investigations aimed to elucidate the pathophysiology not only of $\mathrm{KD}$, but also of other inflammatory disorders whose causative factors and mechanisms are still unknown.

\section{DATA AVAILABILITY STATEMENT}

The original contributions presented in the study are included in the article/supplementary materials, further inquiries can be directed to the corresponding author/s.

\section{AUTHOR CONTRIBUTIONS}

All authors listed have made a substantial, direct and intellectual contribution to the work, and approved it for publication.
3. Whittaker E, Bamford A, Kenny J, Kaforou M, Jones CE, Shah P, et al. Clinical characteristics of 58 children with a pediatric inflammatory multisystem syndrome temporally associated with SARS-CoV-2. JAMA. (2020) 324:25969. doi: 10.1001/jama.2020.10369

4. Pouletty M, Borocco C, Ouldali N, Caseris M, Basmaci R, Lachaume $\mathrm{N}$, et al. Paediatric multisystem inflammatory syndrome temporally associated with SARS-CoV-2 mimicking Kawasaki disease (Kawa-COVID-19): a multicentre cohort. Ann Rheum Dis. (2020) 79:999-1006. doi: 10.1136/annrheumdis-2020-217960 
5. Dufort EM, Koumans EH, Chow EJ, Rosenthal EM, Muse A, Rowlands J, et al. Multisystem inflammatory syndrome in children in New York State. N Engl J Med. (2020) 383:347-58. doi: 10.1056/NEJMoa2021756

6. Feldstein LR, Rose EB, Horwitz SM, Collins JP, Newhams MM, Son MBF, et al. Multisystem inflammatory syndrome in U.S. children and adolescents. N Engl J Med. (2020) 383:334-46. doi: 10.1056/NEJMoa2021680

7. Haslak F, Yildiz M, Adrovic A, Sahin S, Barut K, Kasapçopur Ö. A recently explored aspect of the iceberg named COVID-19: multisystem inflammatory syndrome in children (MIS-C). Turk Arch Pediatr. (2021) 56:3-9. doi: 10.5152/TurkArchPediatr.2020.20245

8. Dong Y, Mo X, Hu Y, Qi X, Jiang F, Jiang Z, et al. Epidemiology of COVID-19 among children in China. Pediatrics. (2020) 145:e20200702. doi: 10.1542/peds.2020-0702

9. Parri $\mathrm{N}$, Lenge $\mathrm{M}$, Buonsenso D. Coronavirus Infection in Pediatric Emergency Departments (CONFIDENCE) Research Group. Children with Covid-19 in pediatric emergency departments in Italy. N Engl J Med. (2020) 383:187-90. doi: 10.1056/NEJMc2007617

10. Castagnoli R, Votto M, Licari A, Brambilla I, Bruno R, Perlini S, et al. Severe acute respiratory syndrome coronavirus 2 (SARS-CoV-2) infection in children and adolescents: a systematic review. JAMA Pediatr. (2020) 174:8829. doi: 10.1001/jamapediatrics.2020.1467

11. Levin M. Childhood multisystem inflammatory syndrome - a new challenge in the pandemic. N Engl J Med. (2020) 383:393-5. doi: 10.1056/NEJMe20 23158

12. Koné-Paut I, Cimaz R. Is it Kawasaki shock syndrome, Kawasaki-like disease or pediatric inflammatory multisystem disease? The importance of semantic in the era of COVID-19 pandemic. RMD Open. (2020) 6:e001333. doi: 10.1136/rmdopen-2020-001333

13. Rowley AH. Multisystem inflammatory syndrome in children and kawasaki disease: two different illnesses with overlapping clinical features. J Pediatr. (2020) 224:129-32. doi: 10.1016/j.jpeds.2020.06.057

14. Bautista-Rodriguez C, Sanchez-de-Toledo J, Clark BC, Herberg J, Bajolle F, Randanne PC, et al. Multisystem inflammatory syndrome in children: an international survey. Pediatrics. (2021) 147:e2020024554. doi: 10.1542/peds.2020-024554

15. Shulman ST. Pediatric coronavirus disease-2019-associated multisystem inflammatory syndrome. J Pediatr Infect Dis Soc. (2020) 9:285-6. doi: 10.1093/jpids/piaa062

16. Rowley AH, Shulman ST, Arditi M. Immune pathogenesis of COVID-19related multisystem inflammatory syndrome in children. J Clin Invest. (2020) 130:5619-21. doi: 10.1172/JCI143840

17. Belay ED, Abrams J, Oster ME, Giovanni J, Pierce T, Meng L, et al. Trends in geographic and temporal distribution of US children with multisystem inflammatory syndrome during the COVID-19 pandemic. JAMA Pediatr. (2021) 6:e210630. doi: 10.1001/jamapediatrics.2021.0630

18. Yeung RS, Ferguson PJ. Is multisystem inflammatory syndrome in children on the Kawasaki syndrome spectrum? J Clin Invest. (2020) 130:56814. doi: 10.1172/JCI141718

19. McCrindle BW, Rowley AH, Newburger JW, Burns JC, Bolger AF, Gewitz $M$, et al. Diagnosis, treatment, and long-term management of kawasaki disease: a scientific statement for health professionals from the American Heart Association. Circulation. (2017) 135:e927-99. doi: 10.1161/CIR.0000000000000484

20. Cohen E, Sundel R. Kawasaki disease at 50 years. JAMA Pediatr. (2016) 170:1093-9. doi: 10.1001/jamapediatrics.2016.1446

21. Dionne A, Dahdah N. Myocarditis and Kawasaki disease. Int J Rheum Dis. (2018) 21:45-9. doi: 10.1111/1756-185X.13219

22. Lin KH, Chang SS, Yu CW, Lin SC, Liu SC, Chao HY, et al. Usefulness of natriuretic peptide for the diagnosis of Kawasaki disease: a systematic review and meta-analysis. BMJ Open. (2015) 5:e006703. doi: 10.1136/bmjopen-2014-006703

23. Parthasarathy P, Agarwal A, Chawla K, Tofighi T, Mondal TK. Upcoming biomarkers for the diagnosis of Kawasaki disease: a review. Clin Biochem. (2015) 48:1188-94. doi: 10.1016/j.clinbiochem.2015.02.013

24. Dengler LD, Capparelli EV, Bastian JF, Bradley DJ, Glode MP, Santa S, et al. Cerebrospinal fluid profile in patients with acute Kawasaki disease. Pediatr Infect Dis J. (1998) 17:478-81. doi: 10.1097/00006454-199806000-00008
25. Kanegaye JT, Wilder MS, Molkara D, Frazer JR, Pancheri J, Tremoulet AH, et al. Recognition of a Kawasaki disease shock syndrome. Pediatrics. (2009) 123:e783-9. doi: 10.1542/peds.2008-1871

26. Natoli V, Rosina S, Ravelli A. Is macrophage activation syndrome in Kawasaki disease underrecognized? J Rheumatol. (2020) 48:1624. doi: 10.3899/jrheum.200361

27. Fajgenbaum DC, June CH. Cytokine storm. N Engl J Med. (2020) 383:225573. doi: $10.1056 /$ NEJMra2026131

28. Ouldali N, Toubiana J, Antona D, Javouhey E, Madhi F, Lorrot $M$, et al. Association of intravenous immunoglobulins plus methylprednisolone vs immunoglobulins alone with course of fever in multisystem inflammatory syndrome in children. JAMA. (2021) 325:855-64. doi: 10.1001/jama.2021.0694

29. Marchesi A, Tarissi de Jacobis I, Rigante D, Rimini A, Malorni W, Corsello G, et al. Kawasaki disease: guidelines of Italian Society of Pediatrics, part II - treatment of resistant forms and cardiovascular complications, followup, lifestyle and prevention of cardiovascular risks. Ital J Pediatr. (2018) 44:103. doi: 10.1186/s13052-018-0529-2

30. Koné-Paut I, Tellier S, Belot A, Brochard K, Guitton C, Marie I, et al. Phase II open label study of anakinra in intravenous immunoglobulin-resistant Kawasaki Disease. Arthritis Rheumatol. (2021) 73:151-61. doi: 10.1002/art.41481

31. Reiff DD, Mannion ML, Samuy N, Scalici P, Cron RQ. Distinguishing active pediatric COVID-19 pneumonia from MIS-C. Pediatr Rheumatol. (2021) 19:21. doi: 10.1186/s12969-021-00508-2

32. Cattalini M, Della Paolera S, Zunica F, Bracaglia C, Giangreco M, Verdoni L, et al. Rheumatology Study Group of the Italian Pediatric Society. Defining Kawasaki disease and pediatric inflammatory multisystem syndrome-temporally associated to SARS-CoV-2 infection during SARSCoV-2 epidemic in Italy: results from a national, multicenter survey. Pediatr Rheumatol Online J. (2021) 19:29. doi: 10.1186/s12969-02100511-7

33. Makino N, Nakamura Y, Yashiro M, Ae R, Tsuboi S, Aoyama Y, et al. Descriptive epidemiology of Kawasaki disease in Japan, 2011-2012: from the results of the 22nd nationwide survey. J Epidemiol. (2015) 25:23945. doi: 10.2188/jea.JE20140089

34. Netea MG, Giamarellos-Bourboulis EJ, Domínguez-Andrés J, Curtis N, van Crevel R, van de Veerdonk FL, et al. Trained immunity: a tool for reducing susceptibility to and the severity of SARS-CoV-2 infection. Cell. (2020) 181:969-77. doi: 10.1016/j.cell.2020.04.042

35. Zhou MY, Xie XL, Peng YG, Wu MJ, Deng XZ, Wu Y, et al. From SARS to COVID-19: what we have learned about children infected with COVID-19. Int J Infect Dis. (2020) 96:710-4. doi: 10.1016/j.ijid.2020.04.090

36. Sidiq KR, Sabir DK, Ali SM, Kodzius R. Does early childhood vaccination protect against COVID-19? Front Mol Biosci. (2020) 7:120. doi: $10.3389 /$ fmolb. 2020.00120

37. Zhou P, Yang XL, Wang XG, Hu B, Zhang L, Zhang W, et al. A pneumonia outbreak associated with a new coronavirus of probable bat origin. Nature. (2020) 579:270-3. doi: 10.1038/s41586-020-2951-z

38. Bunyavanich S, Do A, Vicencio A. Nasal gene expression of angiotensinconverting enzyme 2 in children and adults. JAMA. (2020) 323:24279. doi: 10.1001/jama.2020.8707

39. Chen J, Jiang Q, Xia X, Liu K, Yu Z, Tao W, et al. Individual variation of the SARS-CoV-2 receptor ACE2 gene expression and regulation. Aging Cell. (2020) 19:e13168. doi: 10.1111/acel.13168

40. Lingappan K, Karmouty-Quintana H, Davies J, Akkanti B, Harting MT. Understanding the age divide in COVID-19: why are children overwhelmingly spared? Am J Physiol Lung Cell Mol Physiol. (2020) 319:L3944. doi: 10.1152/ajplung.00183.2020

41. Henderson LA, Canna SW, Friedman KG, Gorelik M, Lapidus SK, Bassiri H, et al. American College of Rheumatology clinical guidance for multisystem inflammatory syndrome in children associated with SARS-CoV-2 and hyperinflammation in pediatric COVID-19: version 2. Arthritis Rheumatol. (2020) 73:e13-29. doi: 10.1002/art.41454

42. Esper F, Shapiro ED, Weibel C, Ferguson D, Landry ML, Kahn JS. Association between a novel human coronavirus and Kawasaki disease. J Infect Dis. (2005) 191:499-502. doi: 10.1086/428291 
43. Shirato K, Imada Y, Kawase M, Nakagaki K, Matsuyama S, Taguchi F. Possible involvement of infection with human coronavirus 229E, but not NL63, in Kawasaki disease. J Med Virol. (2014) 86:2146-53. doi: 10.1002/jmv.23950

44. Ravelli A, Martini A. Kawasaki disease or kawasaki syndrome? Ann Rheum Dis. (2020) 79:993-5. doi: 10.1136/annrheumdis-2020-218110

45. Zhou Q, Yang D, Ombrello AK, Zavialov AV, Toro C, Zavialov AV, et al. Early-onset stroke and vasculopathy associated with mutations in ADA2. N Engl J Med. (2014) 370:911-20. doi: 10.1056/NEJMoa13 07361

46. Navon Elkan P, Pierce SB, Segel R, Walsh T, Barash J, Padeh S, et al. Mutant adenosine deaminase 2 in a polyarteritis nodosa vasculopathy. $N$ Engl J Med. (2014) 370:921-31. doi: 10.1056/NEJMoa1307362

47. Zhou Q, Wang H, Schwartz DM, Stoffels M, Park YH, Zhang Y, et al. Loss-of-function mutations in TNFAIP3 leading to A20 haploinsufficiency cause an early-onset autoinflammatory disease. Nat Genet. (2016) 48:6773. doi: 10.1038/ng.3459

48. Watkin LB, Jessen B, Wiszniewski W, Vece TJ, Jan M, Sha Y, et al. COPA mutations impair ER-Golgi transport and cause hereditary autoimmune-mediated lung disease and arthritis. Nat Genet. (2015) 47:654-60. doi: 10.1038/ng.3279

49. Wakil SM, Monies DM, Abouelhoda M, Al-Tassan N, Al-Dusery H, Naim EA, et al. Association of a mutation in LACC1 with a monogenic form of systemic juvenile idiopathic arthritis. Arthritis Rheumatol. (2015) 67:28895. doi: 10.1002/art.38877

50. Malattia C, Martini A. Paediatric-onset systemic lupus erythematosus. Best Pract Res Clin Rheumatol. (2013) 27:351-62. doi: 10.1016/j.berh.2013. 07.007

51. Caorsi R, Penco F, Grossi A, Insalaco A, Omenetti A, Alessio M, et al. ADA2 deficiency (DADA2) as an unrecognised cause of early onset polyarteritis nodosa and stroke: a multicentre national study. Ann Rheum Dis. (2017) 76:1648-56. doi: 10.1136/annrheumdis-2016-210802

52. Weiss PF, Klink AJ, Luan X, Feudtner C. Temporal association of Streptococcus, Staphylococcus, and parainfluenza pediatric hospitalizations and hospitalized cases of Henoch-Schönlein purpura. J Rheumatol. (2010) 37:2587-94. doi: 10.3899/jrheum.100364

53. Lee PY, Day-Lewis M, Henderson LA, Feudtner C. Distinct clinical and immunological features of SARS-CoV-2-induced multisystem inflammatory syndrome in children. J Rheumatol. (2010) 37:2587-94. doi: 10.1172/JCI141113

54. Toubiana J, Cohen JF, Brice J, Poirault C, Bajolle F, Curtis W, et al. Distinctive features of Kawasaki disease following SARS-CoV-2 infection: a controlled study in Paris, France. J Clin Immunol. (2021) 41:52635. doi: 10.1007/s10875-020-00941-0

55. Manthiram K, Preite S, Dedeoglu F, Demir S, Ozen S, Edwards KM, et al. Common genetic susceptibility loci link PFAPA syndrome, Behçet's disease, and recurrent aphthous stomatitis. Proc Natl Acad Sci USA. (2020) 117:1440511. doi: $10.1073 /$ pnas. 2002051117

56. Zhang Q, Bastard P, Liu Z, Le Pen J, Moncada-Velez M, Chen J, et al. Inborn errors of type I IFN immunity in patients with life-threatening COVID-19. Science. (2020) 370:eabd4570. doi: 10.1126/science.abd4570

57. Bastard P, Rosen LB, Zhang Q, Michailidis E, Hoffmann HH, Zhang Y, et al. Autoantibodies against type I IFNs in patients with life-threatening COVID-19. Science. (2020) 370:eabd4585. doi: 10.1126/science.abd4585

58. Consiglio CR, Cotugno N, Sardh F, Pou C, Amodio D, Rodriguez L, et al. The immunology of multisystem inflammatory syndrome in children with COVID-19. Cell. (2020) 183:968-81.e7. doi: 10.1016/j.cell.2020.09.016

59. Kawasaki T. Acute febrile mucocutaneous syndrome with lymphoid involvement with specific desquamation of the fingers and toes in children [in Japanese]. Arerugi. (1967) 16:178-222.

60. Kawasaki T, Kosaki F, Okawa S, Shigematsu I, Yanagawa H. A new infantile acute febrile mucocutaneous lymph node syndrome (MLNS) prevailing in Japan. Pediatrics. (1974) 54:271-6.

Conflict of Interest: The authors declare that the research was conducted in the absence of any commercial or financial relationships that could be construed as a potential conflict of interest.

Copyright (c) 2021 Matucci-Cerinic, Caorsi, Consolaro, Rosina, Civino and Ravelli. This is an open-access article distributed under the terms of the Creative Commons Attribution License (CC BY). The use, distribution or reproduction in other forums is permitted, provided the original author(s) and the copyright owner(s) are credited and that the original publication in this journal is cited, in accordance with accepted academic practice. No use, distribution or reproduction is permitted which does not comply with these terms. 